\title{
Hydrogen sulfide attenuates myocardial fibrosis in diabetic rats through the JAK/STAT signaling pathway
}

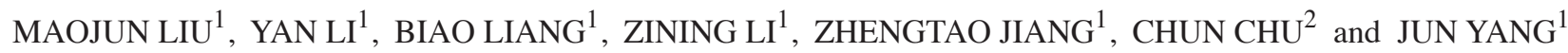 \\ ${ }^{1}$ Department of Cardiology, The First Affiliated Hospital of the University of South China; ${ }^{2}$ Department of Pharmacy, \\ The Second Affiliated Hospital of the University of South China, Hengyang, Hunan 421001, P.R. China
}

Received November 1, 2016; Accepted January 10, 2018

DOI: $10.3892 /$ ijmm.2018.3419

\begin{abstract}
The aim of the present study was to determine the role of hydrogen sulfide $\left(\mathrm{H}_{2} \mathrm{~S}\right)$ in improving myocardial fibrosis and its effects on oxidative stress, endoplasmic reticulum (ER) stress and cell apoptosis in diabetic rats, by regulating the Janus kinase/signal transducer and activator of transcription (JAK/STAT) signaling pathway. A total of 40 male Sprague-Dawley rats were randomly divided into four groups $(\mathrm{n}=10)$ as follows: Normal (control group), diabetes mellitus [streptozotocin (STZ) group], diabetes mellitus treated with $\mathrm{H}_{2} \mathrm{~S}$ (STZ $+\mathrm{H}_{2} \mathrm{~S}$ group), and normal rats treated with $\mathrm{H}_{2} \mathrm{~S}\left(\mathrm{H}_{2} \mathrm{~S}\right.$ group). Diabetes in rats was induced by intraperitoneal (i.p.) injection of STZ at a dose of $40 \mathrm{mg} / \mathrm{kg}$. NaHS $(100 \mu \mathrm{mol} / \mathrm{kg}$, i.p.), which was used as an exogenous donor of $\mathrm{H}_{2} \mathrm{~S}$, was administered to rats in the STZ $+\mathrm{H}_{2} \mathrm{~S}$ and $\mathrm{H}_{2} \mathrm{~S}$ groups. After 8 weeks, the pathological morphological changes in myocardial fibers were observed following hematoxylin and eosin and Masson's trichrome staining. Apoptosis of myocardial tissue was analyzed by the terminal deoxynucleotidyl transferase dUTP nick end labeling assay. Oxidative stress was evaluated through detecting the content of malondialdehyde (MDA), 4-hydroxynonenal (4-HNE), glutathione (GSH) and superoxide dismutase (SOD) in the myocardial cells by ELISA. The expression of collagen III, matrix metalloproteinase (MMP)8, MMP14, tissue inhibitor of metalloproteinase (TIMP)2, transforming growth factor (TGF)- $\beta$, cystathionine- $\gamma$-lyase (CSE), eukaryotic initiation factor $2 \alpha$ (eIF2 $\alpha$ ), GRP94, Bcl-2,
\end{abstract}

Correspondence to: Professor Jun Yang, Department of Cardiology, The First Affiliated Hospital of the University of South China, Hengyang, Hunan 421001, P.R. China

E-mail: yangjunincn@163.com

Abbreviations: $\mathrm{H}_{2} \mathrm{~S}$, hydrogen sulfide; NaHS, sodium hydrosulfide; DCM, diabetic cardiomyopathy; CSE, cystathionine- $\gamma$-lyase; MDA, malondialdehyde; 4-HNE, 4-hydroxynonenal; GSH, glutathione; SOD, superoxide dismutase; STZ, streptozotocin; MMPs, matrix metalloproteinases; CRP, C-reactive protein; eIF2 $\alpha$, eukaryotic initiation factor $2 \alpha$

Key words: hydrogen sulfide, diabetes mellitus, myocardial fibrosis, JAK/STAT signaling pathway, oxidative stress, apoptosis, endoplasmic reticulum stress, inflammatory reaction caspase-3, tumor necrosis factor (TNF)- $\alpha$, nuclear factor- $\kappa \mathrm{B}$ $(\mathrm{NF}-\mathrm{\kappa B})$ and proteins related to the JAK/STAT pathway, was detected by western blot analysis. The results indicated that the array of myocardial cells was markedly disordered in STZ group rats; compared with the control group, both myocardial interstitial fibrosis and the deposition of collagen III were increased. Furthermore, the expression ratio of MMPs/TIMPs was dysregulated, while the expression levels of TGF- $\beta$, eIF2 $\alpha$, GRP94, caspase-3, TNF- $\alpha$, NF- - B, MDA and 4-HNE were significantly increased. Furthermore, the expressions of JAK-1/2 and STAT1/3/5/6 were also markedly upregulated, while those of CSE, SOD, GSH and Bcl-2 were downregulated. Compared with the STZ group, these changes were reversed in the $\mathrm{STZ}+\mathrm{H}_{2} \mathrm{~S}$ group. The results of the present study demonstrated that $\mathrm{H}_{2} \mathrm{~S}$ can improve myocardial fibrosis in diabetic rats, and the underlying mechanism may be associated with the downregulation of the JAK/STAT signaling pathway, thereby suppressing oxidative stress and ER stress, inflammatory reaction and cell apoptosis.

\section{Introduction}

With the improvement of living standards, nutrition-induced diabetes has become a global public health concern, representing the 5th leading cause of mortality worldwide (1). Diabetic cardiomyopathy (DCM) is a major diabetic complication (2). Cardiovascular complications of diabetes are the main cause of hospitalization and death. DCM is an independent complication of diabetes, characterized by early-onset diastolic dysfunction, which is largely attributed to myocardial fibrosis. DCM is a type of cardiomyopathy independent from the large vessels and coronary atherosclerosis, while $75 \%$ of patients with unexplained idiopathic dilated cardiomyopathy have been found to be diabetic (3). DCM is characterized by impaired myocardial insulin signaling, endoplasmic reticulum (ER) stress, mitochondrial dysfunction, activation of the sympathetic nervous system, excessive oxidative stress, increased inflammation, abnormal coronary microcirculation and maladaptive immune responses. These pathophysiological changes result in fibrosis, hypertrophy, cardiac diastolic/systolic dysfunction and, eventually, systolic heart failure. Cardiac interstitial fibrosis is a major characteristic of DCM (4), comprising overproduction and deposition of myocardial interstitial collagen and resulting in myocardial stiffness and cardiac dysfunction. A number of molecular mechanisms 
have been proposed to contribute to the development of DCM, including mechanisms involving oxidative stress, cell apoptosis, autophagy, increased inflammation and ER stress (5-7); however, the exact molecular mechanisms that trigger and drive these major pathological processes have yet to be fully elucidated.

Target organ damage in diabetes mellitus is associated with increased inflammation and oxidative stress, which are considered to be major factors contributing to the development and progression of DCM. The molecular mechanisms regulating related signal conduction remain largely unclear. Previous findings indicate that the activation of Janus kinase/signal transducer and activator of transcription (JAK/STAT) signaling is crucial for the occurrence and development of myocardial fibrosis. The JAK/STAT signaling pathway is a key point in the cytokine signal transduction pathways, as it regulates diverse pathophysiological processes, including proliferation, differentiation, apoptosis, cellular immunity and inflammation (8). Hydrogen sulfide $\left(\mathrm{H}_{2} \mathrm{~S}\right)$ is a colorless, flammable gas with a characteristic odor, which, until recently, had been known to be an endogenously produced gaseous signaling molecule, similar to nitric oxide (NO) and carbon monoxide, and has been implicated in the regulation of inflammatory response, apoptosis, oxidative stress and angiogenesis (9). In addition, $\mathrm{H}_{2} \mathrm{~S}$ has been shown to exert potent cytoprotective effects against tissue injury, including myocardial fibrosis (10). However, the specific mechanism of cardioprotection mediated by $\mathrm{H}_{2} \mathrm{~S}$ in DCM remains largely unknown. There is little evidence on whether the JAK/STAT signaling pathway participates in the protection of exogenous $\mathrm{H}_{2} \mathrm{~S}$ against myocardial fibrosis in diabetes mellitus. In the present study, sodium hydrosulfide (NaHS), an exogenous donor of $\mathrm{H}_{2} \mathrm{~S}$, was used to evaluate the antifibrotic, anti-inflammatory and antioxidant effects of $\mathrm{H}_{2} \mathrm{~S}$ in the hearts of streptozotocin (STZ)-induced diabetic rats. The aim was to provide insight into the molecular mechanisms underlying the action of $\mathrm{H}_{2} \mathrm{~S}$ in myocardial fibrosis associated with diabetes mellitus, improve our understanding of the pathophysiology of DCM, and enable the identification of new therapeutic targets based on the modulation of $\mathrm{H}_{2} \mathrm{~S}$ production.

\section{Materials and methods}

Experimental animals. The experimental protocol was approved by the Animal Ethics Committee of the University of South China (Hengyang, China). A total of 40 adult male Sprague Dawley rats (weight, $300 \pm 20$ g), which were provided by the SJA Animal Experimental Center of Changsha (Changsha, China), were bred in subcages in a clean laboratory with artificial lighting (12-h light/dark cycles), with free access to food and water.

Chemicals and reagents. NaHS was purchased from Sigma-Aldrich; Merck KGaA (St. Louis, MO, USA). STZ was purchased from MP Biomedicals, LLC (Santa Ana, CA, USA). Rabbit polyclonal anti-JAK-1 (cat. no. A00330), rabbit polyclonal anti-JAK-2 (cat. no. BA3398), rabbit polyclonal anti-collagen III (cat. no. BA0326), rabbit polyclonal anti-transforming growth factor (TGF)- $\beta$ (cat. no. BA0290), rabbit polyclonal antitumor necrosis factor (TNF)- $\alpha$ (cat. no. BA14903), rabbit polyclonal anti-nuclear factor (NF)- $\kappa$ B (cat. no. BM3946), mouse anti-STAT1 (cat. no. BA0619-2), mouse anti-STAT3 (cat. no. BA0621), mouse
anti-STAT5 (cat. no.BA1411), mouse anti-STAT6 (cat.no. BA1414), mouse anti-cystathionine- $\gamma$-lyase (CSE; cat. no. BA3605), mouse anti-tissue inhibitor of metalloproteinase (TIMP)2 (cat. no. BA0576), mouse anti-matrix metalloproteinase (MMP)8 (cat. no. BA2201), mouse anti-MMP14 (cat. no. BA1278) and rabbit polyclonal anti-glyceraldehyde 3-phosphate dehydrogenase (GAPDH; cat.no. BM3874), were all purchased from Wuhan Boster Biological Technology, Ltd. (Wuhan, China). The dilution ratio of these antibodies was 1:400. Furthermore, rabbit anti-eukaryotic initiation factor $2 \alpha$ (eIF2 $\alpha$; cat. no. 11233-1-AP), mouse anti-Bcl-2 (cat. no. 12789-1-AP), mouse anti-caspase-3 (cat. no. 19677-1-AP) and mouse anti-GRP94 (cat. no. 14700-1-AP) were all purchased from Proteintech Group, Inc. (Chicago, IL, USA). The dilution ratio of these antibodies was 1:1,000. Anti-rabbit secondary antibodies (cat. no. SA00001-2) were also purchased from Proteintech Group, Inc. The dilution ratio was 1:2,000. Cell lysis buffer for western blot analysis, the ELISA kit of superoxide dismutase (SOD), malondialdehyde (MDA), 4-hydroxynonenal (4-HNE), glutathione (GSH), the bicinchoninic acid (BCA) protein assay kit, the enhanced chemiluminescence reagent kit and the SDS-PAGE gel preparation kit were all obtained from Beyotime Institute of Biotechnology (Shanghai, China).

Model establishment and grouping. A total of 40 experimental animals were randomly divided into four groups $(n=10)$ as follows: Normal (control group), diabetes mellitus (STZ group), diabetes mellitus treated with $\mathrm{H}_{2} \mathrm{~S}$ ( $\mathrm{STZ}+\mathrm{H}_{2} \mathrm{~S}$ group), and normal rats treated with $\mathrm{H}_{2} \mathrm{~S}\left(\mathrm{H}_{2} \mathrm{~S}\right.$ group). The $\mathrm{STZ}$ and $\mathrm{STZ}+\mathrm{H}_{2} \mathrm{~S}$ groups were administered intraperitoneal (i.p.) injections of STZ (40 mg/kg). During the same time, the rats of the control and $\mathrm{H}_{2} \mathrm{~S}$ groups were treated with saline daily (i.p.). After 3 days of STZ injections, rat blood samples were collected through the caudal vein to measure the blood glucose level. Blood glucose $>16.7 \mathrm{mmol} / 1$ suggested successful establishment of the diabetes model. Subsequently, NaHS $(100 \mu \mathrm{mol} / \mathrm{kg}$, i.p.) was administered to the rats of the $\mathrm{STZ}+\mathrm{H}_{2} \mathrm{~S}$ and $\mathrm{H}_{2} \mathrm{~S}$ groups, whereas rats in the control and STZ groups were treated with phosphate-buffered saline daily. The experiment lasted for 8 weeks. The rats were sacrificed following anesthesia with chloral hydrate $(350 \mathrm{mg} / \mathrm{kg})$ and all the animals were weighed. The hearts of the rats were lavaged with ice-cold normal saline before removing and weighing. Three rats were selected randomly from each group. Each heart was divided into two parts, one of which was utilized for terminal deoxynucleotidyl transferase dUTP nick end labeling (TUNEL) assay and the other for biochemical analyses.

Histopathological analysis of myocardial fibers. The removed hearts were fixed in $4 \%$ paraformaldehyde. Each heart was dehydrated with graded alcohols, embedded in paraffin and sliced into 5- $\mu \mathrm{m}$ sections. These sections were stained using a hematoxylin and eosin (H\&E) staining kit and a Masson's trichrome staining kit, and observed under a light microscope at a magnification of $\mathrm{x} 200$.

TUNEL assay. The rat myocardial tissue sections were fixed in $10 \%$ formalin, embedded in paraffin and processed for the TUNEL assay. The slides were treated with $\mathrm{H}_{2} \mathrm{O}_{2}$ and incubated with a reaction mixture containing TdT and digoxigenin-conjugated dUTP for $1 \mathrm{~h}$ at $37^{\circ} \mathrm{C}$. Labeled DNA was visualized 
Table I. Effects of $\mathrm{H}_{2} \mathrm{~S}$ on $\mathrm{BG}$ concentration, BW and $\mathrm{HW}$ in diabetic rats.

\begin{tabular}{lcccc}
\hline Parameters & Control group & STZ group & STZ $+\mathrm{H}_{2} \mathrm{~S}$ group & $\mathrm{H}_{2} \mathrm{~S}$ group \\
\hline $\mathrm{BW}(\mathrm{g})$ & $437.71 \pm 64.75$ & $269.86 \pm 20.41^{\mathrm{b}}$ & $280.14 \pm 12.06^{\mathrm{b}}$ & $471.29 \pm 24.16$ \\
$\mathrm{HW}(\mathrm{g})$ & $1.46 \pm 0.17$ & $1.04 \pm 0.10^{\mathrm{b}}$ & $1.07 \pm 0.13^{\mathrm{b}}$ & $1.52 \pm 0.96^{\mathrm{b}}$ \\
$\mathrm{HW} / \mathrm{BW}(\mathrm{x} 100)$ & $0.34 \pm 0.02$ & $0.39 \pm 0.04^{\mathrm{a}}$ & $0.38 \pm 0.05^{\mathrm{a}}$ & $0.32 \pm 0.01$ \\
$\mathrm{BG}_{1}(\mathrm{mmol} / \mathrm{l})$ & $5.77 \pm 0.33$ & $6.12 \pm 0.83$ & $6.05 \pm 0.63$ & $5.83 \pm 0.77$ \\
$\mathrm{BG}_{2}(\mathrm{mmol} / \mathrm{l})$ & $7.26 \pm 0.58$ & $28.5 \pm 2.55^{\mathrm{b}}$ & $25.2 \pm 5.54^{\mathrm{b}}$ & $8.12 \pm 2.06$ \\
$\mathrm{BG}_{3}(\mathrm{mmol} / \mathrm{l})$ & $6.91 \pm 0.45$ & $26.90 \pm 2.42^{\mathrm{b}}$ & $25.71 \pm 2.38^{\mathrm{b}}$ & $7.32 \pm 0.91$
\end{tabular}

${ }^{\mathrm{a}} \mathrm{P}<0.05$ and ${ }^{\mathrm{b}} \mathrm{P}<0.001$ vs. the control group. Values are expressed as means \pm standard deviation $(\mathrm{n}=7)$. STZ, streptozocin; $\mathrm{H}_{2} \mathrm{~S}$, hydrogen sulfide; $\mathrm{BW}$, body weight; $\mathrm{HW}$, heart weight; $\mathrm{BG}$, blood glucose; $\mathrm{BG}_{1}$, blood glucose prior to the experiment; $\mathrm{BG}_{2}$, blood glucose 3 days after intraperitoneal (i.p.) injection of $\mathrm{STZ} ; \mathrm{BG}_{3}$, blood glucose 8 weeks after i.p. injection of STZ.

with peroxidase-conjugated anti-digoxigenin antibody using 3,3'-diaminobenzidine as the chromogen. Rat testicular tissue was used as positive control in the TUNEL assay.

Measurement of MDA, 4-HNE, GSH and SOD. The content of MDA, 4-HNE and GSH and the activity of SOD in the heart were assayed by ELISA. The ELISA kits for SOD, MDA, 4-HNE and GSH were all obtained from Beyotime Institute of Biotechnology. The steps were conducted following the manufacturer's instructions.

Western blot analysis. Total protein was extracted in ice-cold radioimmunoprecipitation assay buffer containing protease inhibitors (Beyotime Institute of Biotechnology), and quantified using a BCA protein assay kit. Proteins were denatured, separated by SDS-PAGE electrophoresis and transferred to a PVDF membrane by the wet transfer method. The membranes were blocked with 5\% skimmed milk in Tris-buffered saline with Tween-20 (TBST) for $2 \mathrm{~h}$ at room temperature and incubated with blocking solution containing primary antibody (1:400, anti-collagen III; 1:400, anti-MMP8; 1:400, anti-MMP14; 1:400, anti-TIMP2; 1:400, antiCSE; 1:400, anti-TGF- $\beta$; $1: 400$, anti-TNF- $\alpha ; 1: 400$, anti-NF- $\kappa$ B; 1:400, anti-STAT1/3/5/6; 1:400, anti-JAK-1/2; 1:1,000, anti-eIF2 $\alpha$; 1:1,000, anti-GRP94; 1:1,000, anti-caspase-3; and 1:1,000, anti-Bcl-2) overnight at $4^{\circ} \mathrm{C}$. After washing three times with TBST, the membranes were incubated with horseradish peroxidase-conjugated secondary antibody $(1: 2,000)$ for $1 \mathrm{~h}$ at room temperature. Next, the membranes were washed in TBST buffer three times and subjected to chemiluminescence detection assay. The bands were analyzed with a Molecular Imager VersaDoc MP 5000 system (Bio-Rad Laboratories, Inc., Hercules, CA, USA).

Statistical analysis. Data are expressed as mean \pm standard deviation. Statistical differences among the groups were assessed by one-way analysis of variance with SPSS 18.0 software (SPSS Inc., Chicago, IL, USA). Differences between two groups were analyzed using the Student-Newman-Keuls test. $\mathrm{P}<0.05$ was considered to indicate a statistically significant difference.

\section{Results}

Effects of $\mathrm{H}_{2} \mathrm{~S}$ on blood glucose concentration, body weight $(\mathrm{BW})$ and heart weight $(\mathrm{HW})$ in diabetic rats. Plasma

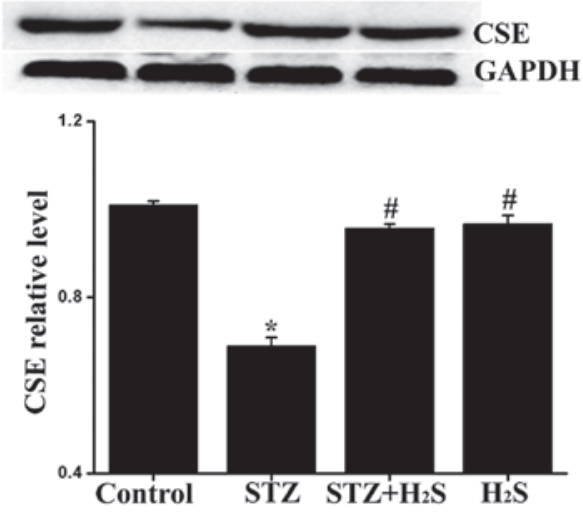

Figure 1. Expression of cystathionine- $\gamma$-lyase (CSE) in each group. Data are expressed as mean \pm standard deviation $(n=3)$. ${ }^{*} \mathrm{P}<0.05$ vs. control group; ${ }^{\#} \mathrm{P}<0.05$ vs. streptozotocin (STZ) group. GAPDH, glyceraldehyde 3-phosphate dehydrogenase.

glucose concentration, BW and $\mathrm{HW}$ were measured prior to sacrificing the rats. The results revealed that BW and $\mathrm{HW}$ in the STZ-treated groups were significantly lower compared with those in the control group. The concentration of blood glucose was significantly increased in the STZ-treated group compared with that in the control group. However, no significant differences in BW, HW or blood glucose concentration were observed between the $\mathrm{H}_{2} \mathrm{~S}$-treated and STZ groups (Table I).

Effects of $\mathrm{H}_{2} \mathrm{~S}$ on CSE expression in diabetic rats. To determine whether diabetes-induced myocardial damage was associated with decreased generation of endogenous $\mathrm{H}_{2} \mathrm{~S}$, the expression level of CSE was measured by western blot analysis. Compared with the control group, the expression level of CSE in the STZ and STZ $+\mathrm{H}_{2} \mathrm{~S}$ groups was significantly decreased, while there was no obvious difference in the $\mathrm{H}_{2} \mathrm{~S}$ group; compared with the STZ group, the myocardial expression of CSE was significantly increased in the $\mathrm{STZ}+\mathrm{H}_{2} \mathrm{~S}$ and $\mathrm{H}_{2} \mathrm{~S}$ groups (Fig. 1).

$\mathrm{H}_{2} \mathrm{~S}$ improves histological changes in rats treated with STZ. On examination under a light microscope, myocardial cells in the control group were orderly and compactly arranged, the intercellular space was normal, and there was less extracellular matrix. Compared with the control group, the cardiomyocytes 


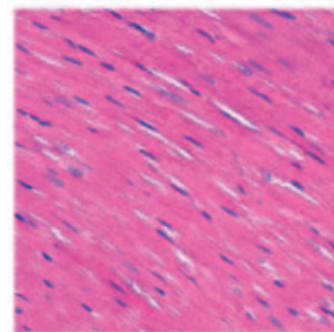

Control

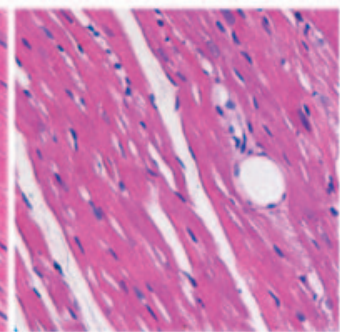

STZ

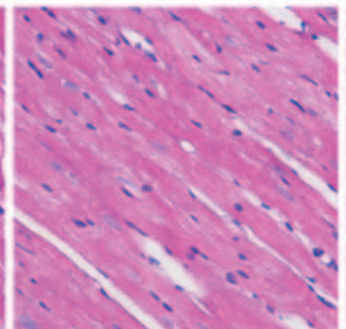

$\mathrm{STZ}+\mathrm{H}_{2} \mathrm{~S}$

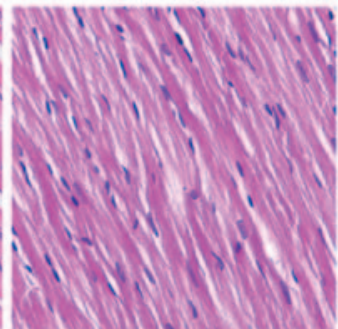

$\mathrm{H}_{2} \mathrm{~S}$

Figure 2. Morphological changes in the myocardium as assessed by hematoxylin and eosin staining (magnification, x200). STZ, streptozocin.

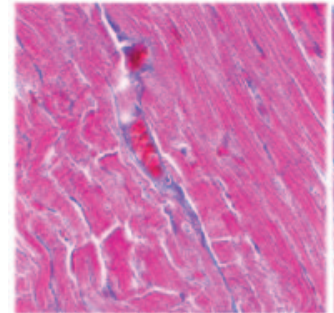

Control

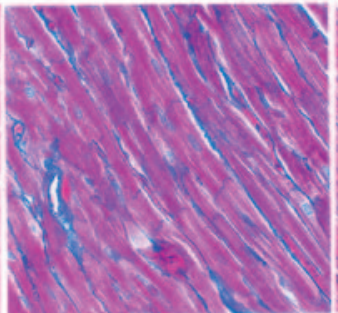

STZ

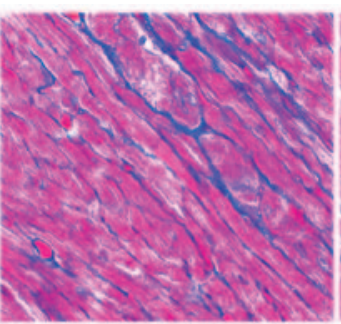

$\mathrm{STZ}+\mathrm{H}_{2} \mathrm{~S}$

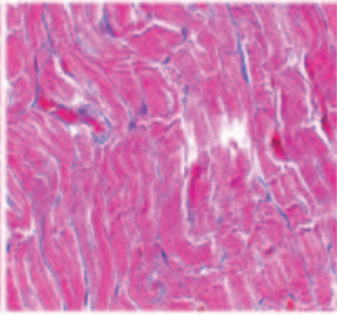

$\mathrm{H}_{2} \mathrm{~S}$

Figure 3. Morphological changes in the myocardium as assessed by Masson's trichrome staining (magnification, x400). STZ, streptozocin.

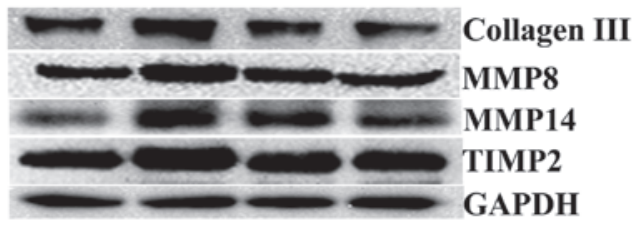

Control STZ STZ+ $\mathrm{H}_{2} \mathrm{~S} \mathrm{H}_{2} \mathrm{~S}$
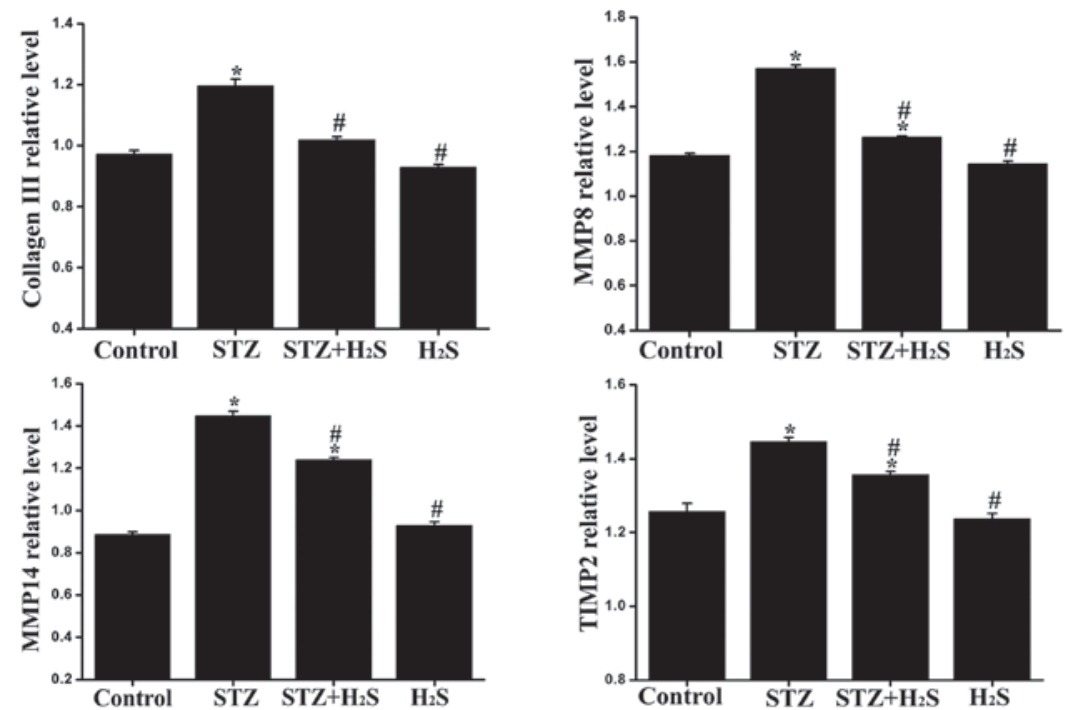

Figure 4. Expression levels of collagen III, matrix metalloproteinase (MMP)8, MMP14 and tissue inhibitor of metalloproteinase (TIMP)2 in each group. Data are expressed as mean \pm standard deviation $(n=3)$. ${ }^{*} \mathrm{P}<0.05$ vs. control group; ${ }^{*} \mathrm{P}<0.05$ vs. streptozotocin (STZ) group. GAPDH, glyceraldehyde 3 -phosphate dehydrogenase.

in the STZ group were disordered, the intercellular spaces were broader, and interstitial fibrosis was present. However, these changes were markedly reversed in the $\mathrm{STZ}+\mathrm{H}_{2} \mathrm{~S}$ group. In the $\mathrm{H}_{2} \mathrm{~S}$ group, the myocardial tissue structure and arrangement of fibers were similar to those of the control group (Fig. 2). Masson's staining revealed the deposition of collagen fibers (blue staining), reflecting the extent of myocardial fibrosis. As demonstrated by Masson's staining, there was little evidence of myocardial fibrosis in the control group. However, a significantly increased degree of fibrosis was observed in the STZ group. Compared with the STZ group, myocardial fibrosis was markedly improved in the STZ $+\mathrm{H}_{2} \mathrm{~S}$ group; however, compared with the control group, there were no significant histological changes in the $\mathrm{H}_{2} \mathrm{~S}$ group (Fig. 3). 


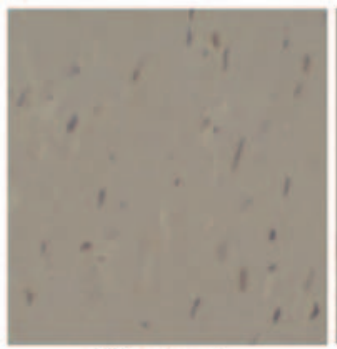

Control

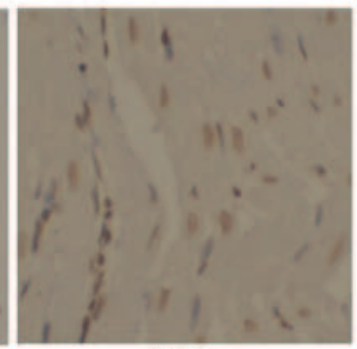

STZ

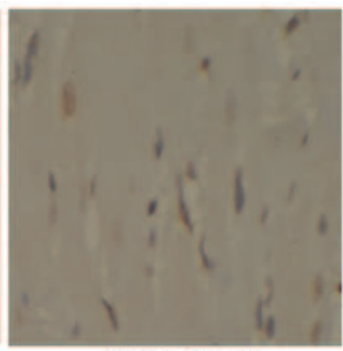

$\mathrm{STZ}+\mathrm{H}_{2} \mathrm{~S}$

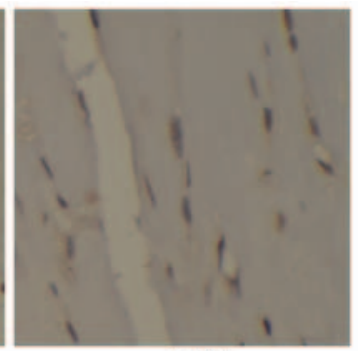

$\mathrm{H}_{2} \mathrm{~S}$

Figure 5. Myocardial cells of heart tissue were detected by the TUNEL assay. Images were captured at magnification of $\mathrm{x} 400$.
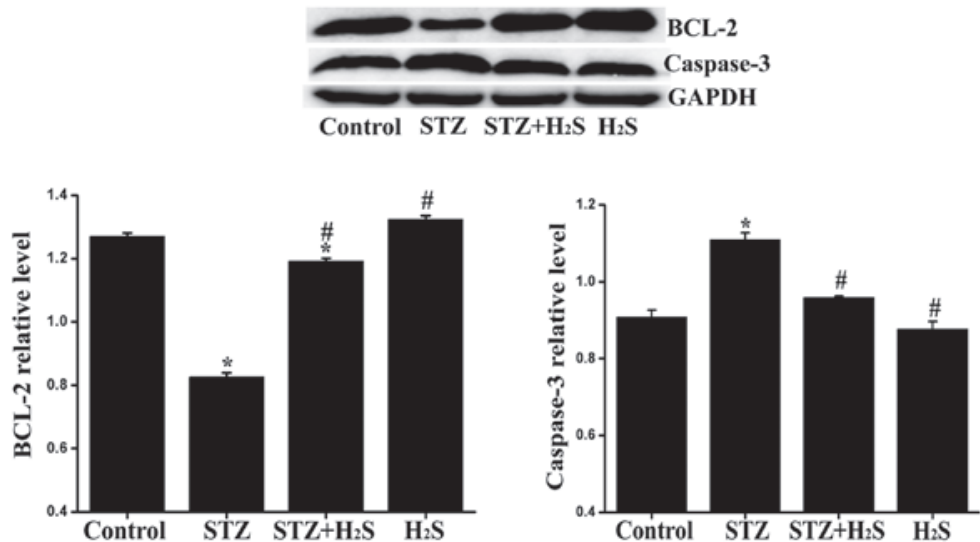

Figure 6. Expression levels of caspase-3 and Bcl-2 in each group. Data are expressed as mean \pm standard deviation ( $=3$ ). ${ }^{*} \mathrm{P}<0.05$ vs. control group; ${ }^{*} \mathrm{P}<0.05$ vs. streptozotocin (STZ) group.

Effects of $\mathrm{H}_{2} \mathrm{~S}$ on collagen III, MMP8, MMP14 and TIMP2 expression in diabetic rats. As the balance of MMPs/TIMPs determines the ratio of collagen synthesis and degradation, it may reflect the status of fibrosis to a certain extent. Therefore, the expression of collagen III, MMP8, MMP14 and TIMP2 was determined. Compared with the control group, the expression levels of collagen III, MMP8, MMP14 and TIMP2 were significantly increased in the STZ group, and the expression levels of TIMP2, MMP8 and MMP14 were significantly increased in the $\mathrm{STZ}+\mathrm{H}_{2} \mathrm{~S}$ group. Compared with the STZ group, the myocardial expression of collagen III, MMP8, MMP14 and TIMP2 was significantly reduced in the $\mathrm{STZ}+\mathrm{H}_{2} \mathrm{~S}$ group. No significant difference was observed in the expression levels of collagen III, MMP8, MMP14 and TIMP2 between the control and $\mathrm{H}_{2} \mathrm{~S}$ groups (Fig. 4).

$\mathrm{H}_{2} \mathrm{~S}$ reduces cardiomyocyte apoptosis in diabetic rats. In the present study, the TUNEL assay was used to detect apoptosis in heart tissue. The number of apoptotic cells was obviously higher in the STZ group compared with that in the control group. However, the number of TUNEL-positive cells was found to be decreased in the $\mathrm{STZ}+\mathrm{H}_{2} \mathrm{~S}$ group. No significant difference was observed between the control and $\mathrm{H}_{2} \mathrm{~S}$ groups (Fig. 5).

Effects of $\mathrm{H}_{2} \mathrm{~S}$ on Bcl-2 and caspase-3 expression in diabetic rats. It is widely accepted that Bcl-2 and caspase- 3 are closely associated with apoptosis. In our experiment, the expression of Bcl-2 and caspase-3 was determined by western blot analysis. Compared with the control group, the expression level of caspase-3 was significantly increased in the STZ group, and the expression level of Bcl-2 was significantly decreased in the $\mathrm{STZ}$ and $\mathrm{STZ}+\mathrm{H}_{2} \mathrm{~S}$ groups. Compared with the STZ group, the myocardial expression of caspase-3 was significantly reduced, whereas that of $\mathrm{Bcl}-2$ was significantly increased in the $\mathrm{STZ}+\mathrm{H}_{2} \mathrm{~S}$ and $\mathrm{H}_{2} \mathrm{~S}$ groups. No significant difference was observed in the expression levels of $\mathrm{Bcl}-2$ and caspase- 3 between the control and $\mathrm{H}_{2} \mathrm{~S}$ groups (Fig. 6).

Protein expression levels of MDA, 4-HNE, GSH and SOD. The levels of GSH, SOD, 4-HNE and MDA were measured to assess oxidative damage in the myocardium and determine whether $\mathrm{H}_{2} \mathrm{~S}$ protects against this type of damage. Compared with the control group, the expression levels of SOD and GSH were significantly decreased in the STZ group, whereas the expression level of SOD was significantly decreased and that of GSH was significantly increased in the $\mathrm{STZ}+\mathrm{H}_{2} \mathrm{~S}$ group (Fig. 7A and B). Compared with the STZ group, the myocardial expression of SOD and GSH was significantly increased in the $\mathrm{STZ}+\mathrm{H}_{2} \mathrm{~S}$ and $\mathrm{H}_{2} \mathrm{~S}$ groups. In addition, the levels of MDA and 4-HNE in STZ group rats were obviously higher compared with those in the control group. Compared with the STZ group, the myocardial expression of MDA and 4-HNE was significantly reduced in the $\mathrm{STZ}+\mathrm{H}_{2} \mathrm{~S}$ and $\mathrm{H}_{2} \mathrm{~S}$ groups (Fig. 7C and D). No significant difference was observed in the expression levels of SOD, GSH, MDA and 4-HNE between the control and $\mathrm{H}_{2} \mathrm{~S}$ groups.

Effects of $\mathrm{H}_{2} \mathrm{~S}$ on TGF- $\beta$ expression in diabetic rats. It is widely accepted that TGF- $\beta$ is closely associated with fibrogenesis. 
A

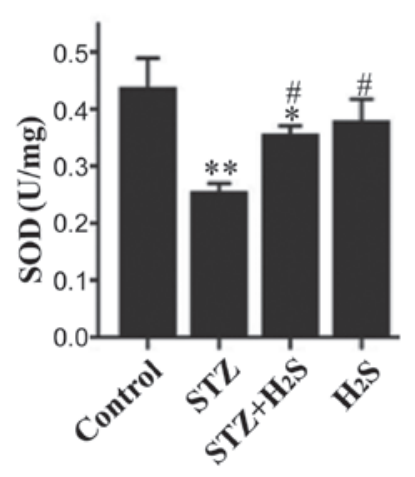

C

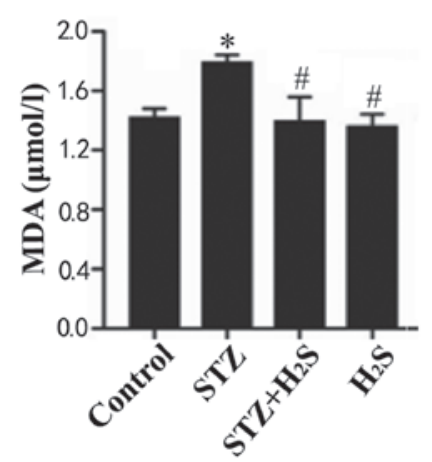

B

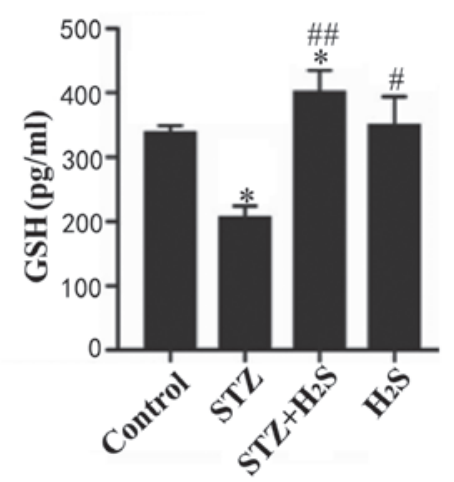

D

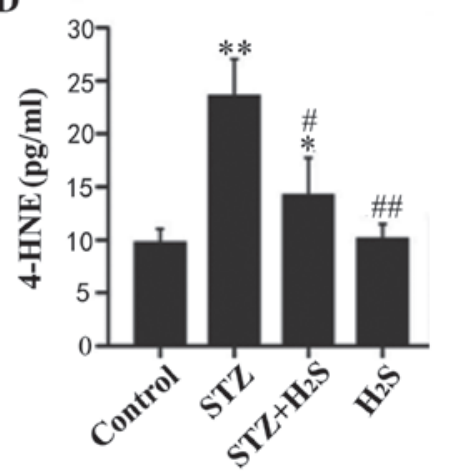

Figure 7. Protein expression of (A) superoxide dismutase (SOD), (B) glutathione (GSH), (C) malondialdehyde (MDA) and (D) 4-hydroxynonenal (4-HNE) in the heart tissue of each group. Data are expressed as mean \pm standard deviation $(\mathrm{n}=3)$. ${ }^{*} \mathrm{P}<0.05$ or ${ }^{* *} \mathrm{P}<0.01$ vs. control group; ${ }^{*} \mathrm{P}<0.05$ or ${ }^{\# \#} \mathrm{P}<0.01$ vs. streptozotocin $(\mathrm{STZ})$ group.

In our experiment, the expression of TGF- $\beta$ was determined by western blot analysis. Compared with the control group, the expression level of TGF- $\beta$ was significantly higher in the STZ group and significantly lower in the $\mathrm{STZ}+\mathrm{H}_{2} \mathrm{~S}$ group, while there was no obvious difference between the control and $\mathrm{H}_{2} \mathrm{~S}$ groups. Compared with the STZ group, the myocardial expression of TGF- $\beta$ was significantly reduced in the $\mathrm{STZ}+\mathrm{H}_{2} \mathrm{~S}$ group (Fig. 8).

Effects of $\mathrm{H}_{2} \mathrm{~S}$ on $T N F-\alpha$ and $N F-\kappa B$ expression in diabetic rats. The expression levels of TNF- $\alpha$ and NF- $\kappa \mathrm{B}$ were measured to assess inflammatory response using western blot analysis. Compared with the control group, the expression levels of TNF- $\alpha$ and NF- $\kappa \mathrm{B}$ were significantly increased in the STZ group, the expression level of TNF- $\alpha$ was significantly decreased in the STZ $+\mathrm{H}_{2} \mathrm{~S}$ group, while there was no significant difference between the control and $\mathrm{H}_{2} \mathrm{~S}$ groups. Compared with the STZ group, the myocardial expression of TNF- $\alpha$ and NF- $\kappa \mathrm{B}$ were significantly reduced in the $\mathrm{STZ}+\mathrm{H}_{2} \mathrm{~S}$ group (Fig. 9).

Effects of $\mathrm{H}_{2} \mathrm{~S}$ on eIF $2 \alpha$ and GRP94 expression in diabetic rats. The expression of eIF $2 \alpha$ and GRP94 was determined by western blot analysis to assess ER stress in the myocardium. Compared with the control group, the expression levels of eIF2 $\alpha$ and GRP94 were significantly increased in the STZ group and the expression of GRP94 was significantly increased in the $\mathrm{STZ}+\mathrm{H}_{2} \mathrm{~S}$ group. Compared with the STZ group, the myocardial expression of eIF2 $\alpha$ and GRP94 was significantly reduced in the $\mathrm{STZ}+\mathrm{H}_{2} \mathrm{~S}$ group. No significant difference

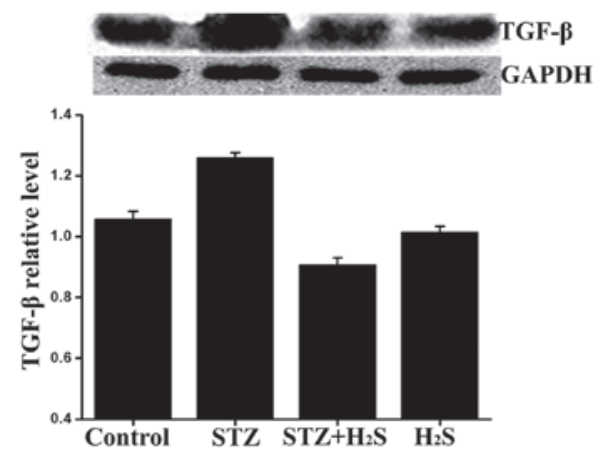

Figure 8. Expression level of transforming growth factor- $\beta$ (TGF- $\beta$ ) in each group. Data are expressed as mean \pm standard deviation $(n=3)$. ${ }^{*} \mathrm{P}<0.05$ vs. control group; ${ }^{\sharp} \mathrm{P}<0.05$ vs. streptozotocin (STZ) group. GAPDH, glyceraldehyde 3-phosphate dehydrogenase.

was observed in the expression levels of eIF2 $\alpha$ and GRP94 between the control and $\mathrm{H}_{2} \mathrm{~S}$ groups (Fig. 10).

Administration of exogenous $\mathrm{H}_{2} \mathrm{~S}$ donor affects JAK-1/2 and STAT1/3/5/6 signaling. To further explore the potential signaling pathway involved in diabetes, proteins associated with the JAK/STAT pathway were detected by western blot analysis. Compared with the control group, the expression level of JAK-1/2 in the STZ group was markedly increased, the expression level of JAK-1 in the $\mathrm{STZ}+\mathrm{H}_{2} \mathrm{~S}$ group was significantly decreased, and the expression levels of JAK-2 in $\mathrm{STZ}+\mathrm{H}_{2} \mathrm{~S}$ group was significantly increased. Compared with the STZ group, the myocardial expression of JAK-1/2 

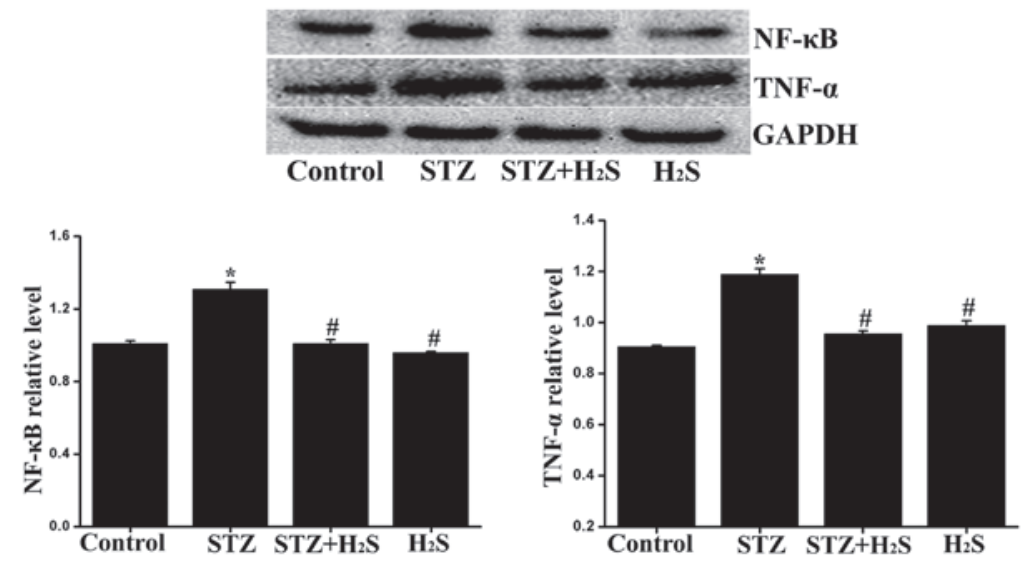

Figure 9. Expression levels of tumor necrosis factor (TNF)- $\alpha$ and nuclear factor (NF)- $\mathrm{\kappa B}$ in each group. Data are expressed as mean \pm standard deviation $(\mathrm{n}=3$ ). ${ }^{*} \mathrm{P}<0.05$ vs. control group; ${ }^{~} \mathrm{P}<0.05$ vs. streptozotocin (STZ) group. GAPDH, glyceraldehyde 3-phosphate dehydrogenase.
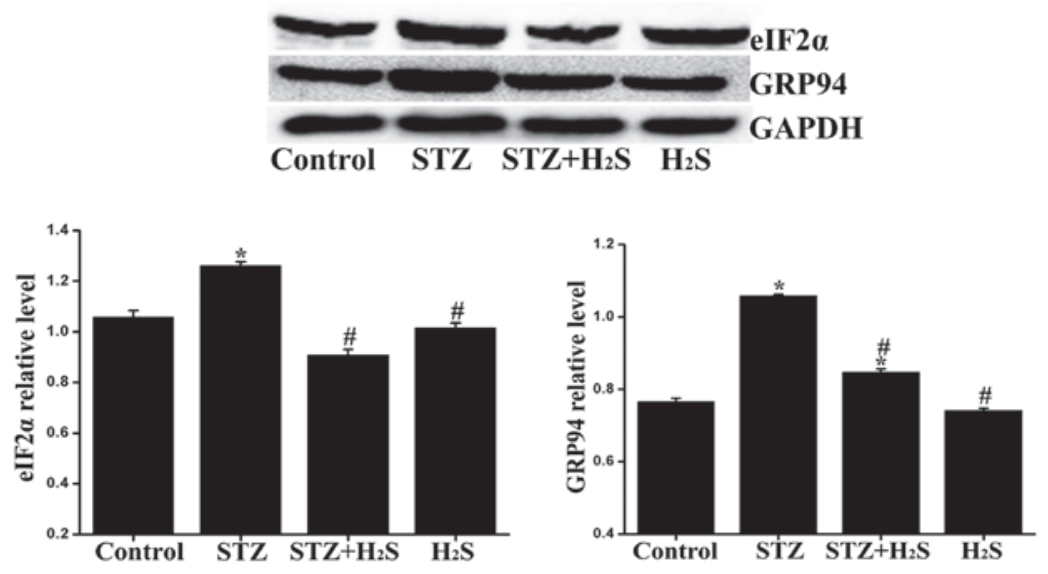

Figure 10. The expression levels of eukaryotic initiation factor $2 \alpha$ (eIF2 $\alpha$ ) and GRP94 in each group. Data are expressed as mean \pm standard deviation (n=3). ${ }^{\text {*P }}<0.05$ vs. control group; ${ }^{*} \mathrm{P}<0.05$ vs. streptozotocin (STZ) group. GAPDH, glyceraldehyde 3-phosphate dehydrogenase.

exhibited a marked reduction in the $\mathrm{STZ}+\mathrm{H}_{2} \mathrm{~S}$ group. No significant difference was observed in the expression levels of JAK-1 and -2 between the control and $\mathrm{H}_{2} \mathrm{~S}$ groups (Fig. 11). Compared with the control group, the expression levels of STAT1/3/5/6 in the STZ group were significantly increased. Compared with the STZ group, the myocardial expression of STAT1/3/5/6 was significantly reduced in the $\mathrm{STZ}+\mathrm{H}_{2} \mathrm{~S}$ group. No significant difference was observed in the expression levels of STAT1/3/5/6 between the control and $\mathrm{H}_{2} \mathrm{~S}$ groups (Fig. 12).

\section{Discussion}

Over the past two decades, the worldwide incidence of diabetes mellitus has steadily increased as a consequence of higher rates of obesity and changes in lifestyle. If unaddressed, nearly half a billion individuals will suffer from diabetes mellitus in 2030 (11). Diabetes is associated with a number of fatal complications, such as DCM, which accounts for most of the morbidity and mortality in this population and represents a major global health concern (12).

Cardiac interstitial fibrosis, as a major characteristic of DCM, results from the overproduction and deposition of myocardial interstitial collagen, leading to hypertrophy, myocardial stiffness, cardiac diastolic/systolic dysfunction and, eventually, heart failure (13). The results of immunohistochemical analysis revealed obvious interstitial fibrosis in the myocardium of diabetic rats. Compared with the control group, the arrangement of myocardial cells was markedly disordered, and collagen deposition in cardiac extracellular matrix was increased in the STZ group. The expression of collagen III in the myocardium was also significantly increased in the STZ group. The results of H\&E and Masson's staining in the present study also demonstrated that diabetes increased the relative disorganization of myocardial cells and enhanced deposition of collagen in the STZ group, clearly indicating that myocardial damage was caused by diabetes. Furthermore, hyperglycemia induced an increase in the heart-to-body weight (HW/BW) ratio. In addition, the results of the present study demonstrated that there was a deregulation of MMPs/TIMPs expression in the myocardial tissue of diabetic rats. All the abovementioned results suggest that there was obvious cardiac interstitial fibrosis in diabetic rats.

Numerous mechanisms may collectively contribute to the development of DCM, including oxidative stress, insulin resistance, myocardial inflammation and ER stress $(14,15)$. However, the exact molecular mechanisms that trigger and fuel these major pathological processes are not entirely clear. An 

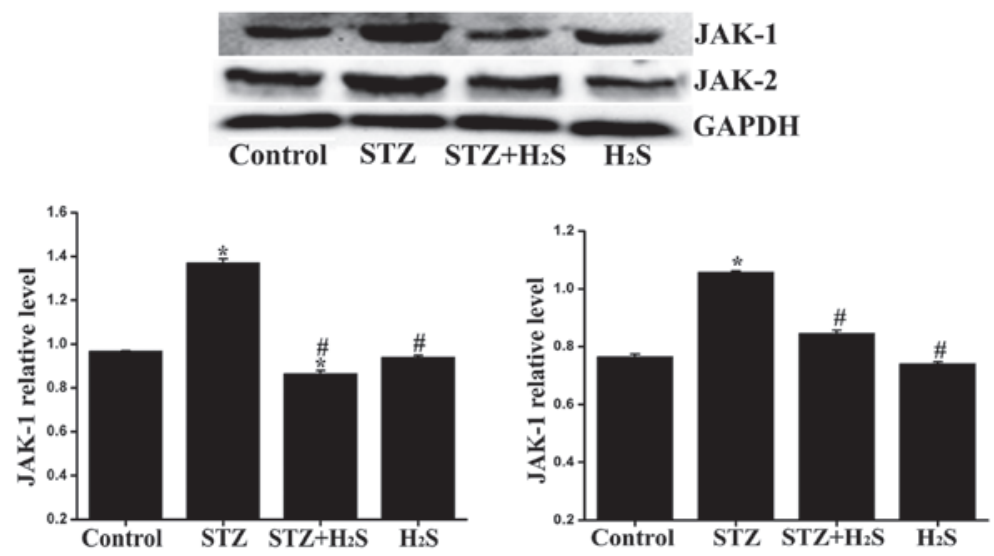

Figure 11. Expression levels of Janus kinase (JAK)-1 and -2 in each group. Data are expressed as mean \pm standard deviation ( $\mathrm{n}=3$ ). "P<0.05 vs. control group; ${ }^{\text {"P }}<0.05$ vs. streptozotocin (STZ) group. GAPDH, glyceraldehyde 3-phosphate dehydrogenase.
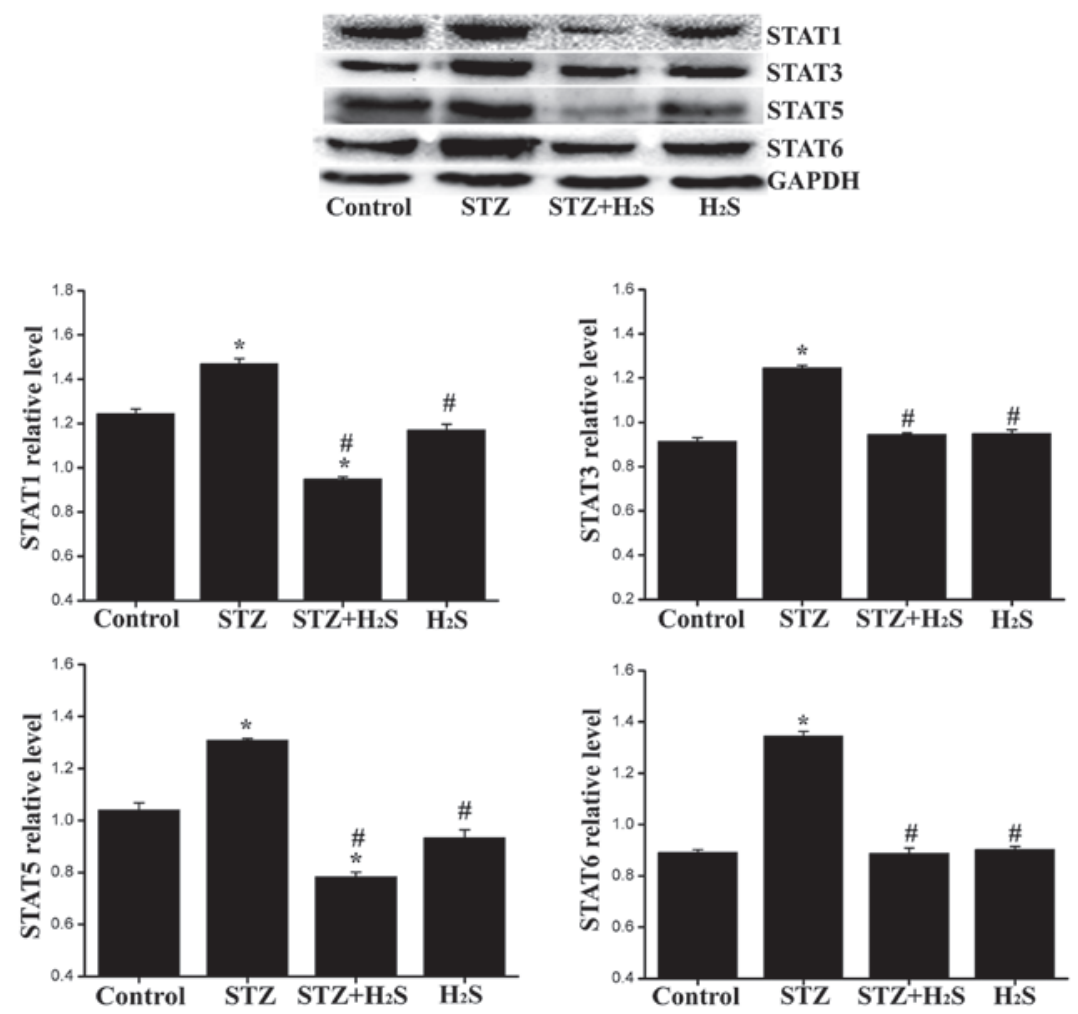

Figure 12. Expression levels of signal transducer and activator of transcription (STAT)1/3/5/6 in each group. Data are expressed as mean \pm standard deviation $(\mathrm{n}=3) .{ }^{*} \mathrm{P}<0.05$ vs. control group; ${ }^{*} \mathrm{P}<0.05$ vs. streptozotocin (STZ) group. GAPDH, glyceraldehyde 3-phosphate dehydrogenase.

increasing number of clinical and experimental studies indicate that sustained hyperglycemia results in reduced antioxidant capacity and increased oxidative stress that are involved in development of diabetes and its complications (16-18). Inflammation is currently recognized as a key contributor to the pathogenesis of diabetes and its cardiovascular complications (19-21), and the diabetic myocardium exhibits increased levels of proinflammatory cytokines, such as TNF- $\alpha$ and IL-6 (22). Increased oxidative stress is another common characteristic in models of DCM. Oxidative stress indicates a severe imbalance between the generation of reactive oxygen species (ROS) and their clearance by antioxidant defense systems, which make the heart highly susceptible to oxidative damage $(23,24)$. ROS may induce lipid peroxidation and result in the increased expression of MDA and 4-HNE. There are natural detoxification molecules in the heart that reduce or scavenge ROS, such as SOD and GSH. ROS, as the main inducer of oxidative stress in vivo, may also result in cell apoptosis. Accordingly, ROS and oxidative stress play an important role in the occurrence and development of diabetic cardiovascular complications $(25,26)$. In the present study, oxidative stress was evaluated through detecting the content of MDA, 4-HNE, GSH and SOD in myocardial cells by ELISA. The expression of collagen III, MMP8, MMP14, TIMP2, TGF- $\beta$, CSE, eIF2 $\alpha$, GRP94, Bcl-2, caspase-3, TNF- $\alpha$, $\mathrm{NF}-\kappa \mathrm{B}, \mathrm{JAK}-1 / 2$ and STAT1/3/5/6 was analyzed by western blotting and the results demonstrated that the expression of MDA, 4-HNE, TNF- $\alpha$, NF- $\kappa$ B, TGF- $\beta$, eIF2 $\alpha$, GRP9 4 and caspase-3 was significantly increased in the STZ group, and the 
expression of SOD, GSH and Bcl-2 was markedly decreased in the STZ group. These results suggest that there was significantly increased oxidative stress in the myocardium of diabetic rats. Furthermore, there was overactivation of ER stress pathways. Inflammatory factors, such as TNF- $\alpha, N F-\kappa B$ and TGF- $\beta$, were also significantly increased. In the STZ group, the extent of myocardial tissue damage gradually increased, along with a significantly increased apoptotic cell rate, demonstrating that cells failed to repair themselves. The result of the TUNEL assay revealed that, compared with the control group, there was a significantly increased number of apoptotic cells in the myocardium of diabetic rats. The expression of caspase- 3 was significantly upregulated in the myocardium of diabetic rats, while that of Bcl-2 was significantly downregulated.

ER stress is an adaptive response of cells to ischemia, hypoxia and hyperglycemia $(27,28)$. Oxidative stress may lead to ER stress (29), and GRP94 and eIF2 $\alpha$ are classic markers of ER stress. Consistent with previous studies $(30,31)$, the expression of GRP94 and eIF2 $\alpha$ in the myocardium of diabetic rats were obviously upregulated in the present study, suggesting the presence of excessive ER stress. TGF- $\beta 1$ is currently considered as one of the most important factors promoting myocardial fibrosis and is considered to play an important role in this process (32). It has been suggested that TGF- $\beta 1$ plays a key role in myocardial interstitial fibrosis in DCM (33). In our experiments, the TGF- $\beta 1$ expression in the myocardium of rats with DCM was significantly higher compared with that in control rats, and was positively correlated with the content of collagen; thus, TGF- $\beta$ may be involved in the regulation and crosstalk of oxidative stress, ER stress, inflammation and apoptosis of cardiomyocytes.

The JAK/STAT signaling pathway is an important cytokine signal transduction pathway and a pleiotropic cascade that is crucial for cytokine and growth hormone receptor signaling, and regulates diverse physiological and pathological processes, including proliferation, differentiation, apoptosis and inflammation (34). In the present study, the expression of GRP94 and eIF $2 \alpha$ in the myocardium of diabetic rats were found to be obviously upregulated, suggesting the presence of excessive ER stress. The activation of the JAK/STAT signal transduction pathway may upregulate the expression of TGF- $\beta$ and type I and III collagens, leading to the occurrence of fibrosis $(35,36)$. It was recently demonstrated that the exposure of glomerular mesangial cells to high glucose caused the activation of JAK-2, STAT1, STAT3 and STAT5, along with an increase in TGF- $\beta 1$ and fibronectin synthesis (37). Conversely, it was reported that inhibiting JAK/STAT signaling in mesangial cells cultured in high glucose may decrease the synthesis of TGF- $\beta 1$ and fibrin (38). The JAK/STATs signal transduction pathway is closely associated with myocardial fibrosis and cardiac hypertrophy caused by pressure overload, heart failure and cardiac dysfunction induced by ischemia-reperfusion (8). In our study, the expression levels of JAK-1, JAK-2 and STATs were significantly upregulated in rats of the STZ group compared with control rats, and the relative contents of JAK-1/2 and STATs were positively correlated with the content of collagen and the expression of TGF- $\beta 1$ in the myocardium. It is suggested that high glucose or glycosylated products in diabetes induce oxidative stress to produce inflammatory cytokines followed by the activation of JAK/STAT and TGF- $\beta$ signaling.
$\mathrm{H}_{2} \mathrm{~S}$ is an endogenously produced gaseous molecule that plays an important role in cellular signaling and possesses potent anti-inflammatory, antioxidant, and other regulatory properties $(39,40)$. In addition, $\mathrm{H}_{2} \mathrm{~S}$ has been shown to exert potent cytoprotective effects against tissue injury as well as antifibrotic effects, including prevention of myocardial fibrosis (41). The reported cytoprotective effects of $\mathrm{H}_{2} \mathrm{~S}$ are partially associated with its ability to neutralize ROS, reduce apoptotic signaling, and reversibly modulate mitochondrial respiration (42). However, the association between $\mathrm{H}_{2} \mathrm{~S}$ and JAK/STAT signaling remains unclear. Our results demonstrated that the expression level of CSE was significantly downregulated in rats of the DCM group compared with control rats, suggesting that diabetes impairs the expression of endogenous $\mathrm{H}_{2} \mathrm{~S} / \mathrm{CSE}$. It was observed that, compared with the rats in the STZ group, the expression of MDA, 4-HNE, TNF- $\alpha$, NF- $\kappa$ B, TGF- $\beta$, eIF $2 \alpha$, GRP9 4 and caspase-3 was significantly downregulated and the expression of SOD, GSH and Bcl-2 was significantly higher in the myocardium of rats treated with the $\mathrm{H}_{2} \mathrm{~S}$ donor NaHS. The levels of JAK- $1 / 2$ and STATs were lower, the expression of TGF- $\beta 1$ was downregulated, the content of collagen in the myocardium decreased, and the level of myocardial fibrosis was visibly reduced in the myocardium of rats in the $\mathrm{STZ}+\mathrm{H}_{2} \mathrm{~S}$ group. $\mathrm{H}_{2} \mathrm{~S}$ was able to attenuate matrix deposition and myocardial fibrosis, reduce apoptosis, alleviate inflammation, improve the deregulation of MMPs/TIMPs, and inhibit excessive oxidative stress and ER stress in the myocardium of diabetic rats. The protective mechanism of $\mathrm{H}_{2} \mathrm{~S}$ against diabetic myocardial fibrosis may be associated with the downregulation of JAK/STAT and TGF- $\beta 1$ signaling. These findings indicate that $\mathrm{H}_{2} \mathrm{~S}$ exerts a protective effect against myocardial interstitial fibrosis in DCM by downregulating JAK/STAT signaling.

Moreover, these results suggest that $\mathrm{H}_{2} \mathrm{~S}$, acts as a scavenger of ROS, enhances the endogenous antioxidant defenses and creates an environment resistant to oxidative stress in the myocardium of diabetic rats. Another major finding of the present study is that the JAK/STAT signaling pathway plays an important role in mediating the cardioprotective effects of $\mathrm{H}_{2} \mathrm{~S}$. To date, it has not been fully elucidated how $\mathrm{H}_{2} \mathrm{~S}$ exerts its beneficial effect against cardiac fibrosis under diabetic conditions via the JAK/STAT signaling pathway. However, as $\mathrm{H}_{2} \mathrm{~S}$ attenuates diabetes-induced oxidative damage and the subsequent cardiac fibrosis, it appears to be a promising novel therapeutic strategy for the prevention and treatment of DCM.

\section{Acknowledgements}

The present study was supported by the National Natural Science Foundation of China (grant nos. 81202830 and 81270181).

\section{Competing interests}

The authors declare that they have no competing interests.

\section{References}

1. Yang W, Lu J, Weng J, Jia W, Ji L, Xiao J, Shan Z, Liu J, Tian H, Ji Q, et al; China National Diabetes and Metabolic Disorders Study Group: Prevalence of diabetes among men and women in China. N Engl J Med 362: 1090-1101, 2010. 
2. Bell DS: Diabetic cardiomyopathy. Diabetes Care 26: 2949-2951, 2003.

3. Asbun $\mathbf{J}$ and Villarreal FJ: The pathogenesis of myocardial fibrosis in the setting of diabetic cardiomyopathy. J Am Coll Cardiol 47: 693-700, 2006.

4. Bugger $\mathrm{H}$ and Abel ED: Molecular mechanisms of diabetic cardiomyopathy. Diabetologia 57: 660-671, 2014

5. Thandavarayan RA, Giridharan VV, Watanabe K and Konishi T: Diabetic cardiomyopathy and oxidative stress: role of antioxidants. Cardiovasc Hematol Agents Med Chem 9: 225-230, 2011.

6. Kumar S, Prasad S and Sitasawad SL: Multiple antioxidants improve cardiac complications and inhibit cardiac cell death in streptozotocin-induced diabetic rats. PLoS One 8: e67009, 2013.

7. Varga ZV, Giricz Z, Liaudet L, Haskó G, Ferdinandy P and Pacher P: Interplay of oxidative, nitrosative/nitrative stress, inflammation, cell death and autophagy in diabetic cardiomyopathy. Biochim Biophys Acta 1852: 232-242, 2015.

8. Kiu H and Nicholson SE: Biology and significance of the JAK/STAT signalling pathways. Growth Factors 30: 88-106, 2012.

9. Kimura H: Production and physiological effects of hydrogen sulfide. Antioxid Redox Signal 20: 783-793, 2014.

10. Lavu M, Bhushan S and Lefer DJ: Hydrogen sulfide-mediated cardioprotection: mechanisms and therapeutic potential. Clin Sci (Lond) 120: 219-229, 2011.

11. Wild S, Roglic G, Green A, Sicree R and King H: Global prevalence of diabetes: estimates for the year 2000 and projections for 2030. Diabetes Care 27: 1047-1053, 2004.

12. Chen Y, Du J, Zhao YT, Zhang L, Lv G, Zhuang S, Qin G and Zhao TC: Histone deacetylase (HDAC) inhibition improves myocardial function and prevents cardiac remodeling in diabetic mice. Cardiovasc Diabetol 14: 99, 2015.

13. Falcão-Pires I and Leite-Moreira AF: Diabetic cardiomyopathy: understanding the molecular and cellular basis to progress in diagnosis and treatment. Heart Fail Rev 17: 325-344, 2012.

14. Yang L, Zhao D, Ren J and Yang J: Endoplasmic reticulum stress and protein quality control in diabetic cardiomyopathy. Biochim Biophys Acta 1852: 209-218, 2015.

15. Jia G, DeMarco VG and Sowers JR: Insulin resistance and hyperinsulinaemia in diabetic cardiomyopathy. Nat Rev Endocrinol 12 $144-153,2016$

16. Sun X, Chen RC, Yang ZH, Sun GB, Wang M, Ma XJ, Yang LJ and Sun XB: Taxifolin prevents diabetic cardiomyopathy in vivo and in vitro by inhibition of oxidative stress and cell apoptosis Food Chem Toxicol 63: 221-232, 2014.

17. Zhao Y, Zhang L, Qiao Y, Zhou X, Wu G, Wang L, Peng Y, Dong X, Huang H, Si L, et al: Heme oxygenase-1 prevents cardiac dysfunction in streptozotocin-diabetic mice by reducing inflammation, oxidative stress, apoptosis and enhancing autophagy. PLoS One 8: e75927, 2013.

18. Taye A, Abouzied MM and Mohafez OM: Tempol ameliorates cardiac fibrosis in streptozotocin-induced diabetic rats: role of oxidative stress in diabetic cardiomyopathy. Naunyn Schmiedebergs Arch Pharmacol 386: 1071-1080, 2013.

19. Malfitano C, Alba Loureiro TC, Rodrigues B, Sirvente R, Salemi VM, Rabechi NB, Lacchini S, Curi R and Irigoyen MC: Hyperglycaemia protects the heart after myocardial infarction: aspects of programmed cell survival and cell death. Eur J Heart Fail 12: 659-667, 2010.

20. Van Linthout S, Riad A, Dhayat N, Spillmann F, Du J, Dhayat S, Westermann D, Hilfiker-Kleiner D, Noutsias M, Laufs U, et al: Anti-inflammatory effects of atorvastatin improve left ventricular function in experimental diabetic cardiomyopathy. Diabetologia 50: 1977-1986, 2007.

21. Westermann D, Walther T, Savvatis K, Escher F, Sobirey M, Riad A, Bader M, Schultheiss HP and Tschöpe C: Gene deletion of the kinin receptor B1 attenuates cardiac inflammation and fibrosis during the development of experimental diabetic cardiomyopathy. Diabetes 58: 1373-1381, 2009.

22. Westermann D, Van Linthout S, Dhayat S, Dhayat N, Schmidt A, Noutsias M, Song XY, Spillmann F, Riad A, Schultheiss HP, et al: Tumor necrosis factor-alpha antagonism protects from myocardial inflammation and fibrosis in experimental diabetic cardiomyopathy. Basic Res Cardiol 102: 500-507, 2007.

23. Yu W, Zha W, Guo S, Cheng H, Wu J and Liu C: Flos Puerariae extract prevents myocardial apoptosis via attenuation oxidative stress in streptozotocin-induced diabetic mice. PLoS One 9: e98044, 2014.
24. Giacco F and Brownlee M: Oxidative stress and diabetic complications. Circ Res 107: 1058-1070, 2010.

25. Frustaci A, Kajstura J, Chimenti C, Jakoniuk I, Leri A, Maseri A, Nadal-Ginard B and Anversa P: Myocardial cell death in human diabetes. Circ Res 87: 1123-1132, 2000.

26. Yaras N, Sariahmetoglu M, Bilginoglu A, Aydemir-Koksoy A, Onay-Besikci A, Turan B and Schulz R: Protective action of doxycycline against diabetic cardiomyopathy in rats. Br J Pharmacol 155: 1174-1184, 2008.

27. Tajiri S, Oyadomari S, Yano S, Morioka M, Gotoh T, Hamada JI, Ushio Y and Mori M: Ischemia-induced neuronal cell death is mediated by the endoplasmic reticulum stress pathway involving CHOP. Cell Death Differ 11: 403-415, 2004.

28. Williams JA, Hou Y, Ni HM and Ding WX: Role of intracellular calcium in proteasome inhibitor-induced endoplasmic reticulum stress, autophagy, and cell death. Pharm Res 30: 2279-2289, 2013.

29. Tang C, Koulajian K, Schuiki I, Zhang L, Desai T, Ivovic A, Wang P, Robson-Doucette C, Wheeler MB, Minassian B, et al: Glucose-induced beta cell dysfunction in vivo in rats: link between oxidative stress and endoplasmic reticulum stress. Diabetologia 55: 1366-1379, 2012.

30. Miki T, Miura T, Hotta H, Tanno M, Yano T, Sato T, Terashima Y, Takada A, Ishikawa S and Shimamoto K: Endoplasmic reticulum stress in diabetic hearts abolishes erythropoietin-induced myocardial protection by impairment of phospho-glycogen synthase kinase-3beta-mediated suppression of mitochondrial permeability transition. Diabetes 58: 2863-2872, 2009.

31. .Guo R, Liu W, Liu B, Zhang B, Li W and Xu Y: SIRT1 suppresses cardiomyocyte apoptosis in diabetic cardiomyopathy: An insight into endoplasmic reticulum stress response mechanism. Int J Cardiol 191: 36-45, 2015.

32. Zeglinski MR, Roche P, Hnatowich M, Jassal DS, Wigle JT, Czubryt MP and Dixon IM: TGF $\beta 1$ regulates Scleraxis expression in primary cardiac myofibroblasts by a Smad-independent mechanism. Am J Physiol Heart Circ Physiol 310: H239-H249, 2016

33. Liu Y and Zhang J: Nox2 contributes to cardiac fibrosis in diabetic cardiomyopathy in a transforming growth factor- $\beta$ dependent manner. Int J Clin Exp Pathol 8: 10908-10914, 2015.

34. Duhé RJ: Redox regulation of Janus kinase: the elephant in the room. JAKSTAT 2: e26141, 2013.

35. Shi K, Jiang J, Ma T, Xie J, Duan L, Chen R, Song P, Yu Z, Liu C, Zhu Q, et al: Dexamethasone attenuates bleomycin-induced lung fibrosis in mice through TGF- $\beta$, Smad3 and JAK-STAT pathway. Int J Clin Exp Med 7: 2645-2650, 2014.

36. Matsui F and Meldrum KK: The role of the Janus kinase family/signal transducer and activator of transcription signaling pathway in fibrotic renal disease. J Surg Res 178: 339-345, 2012.

37. Shi Y, Zhang Y, Wang C, Du C, Zhao S, Qi Z, Zhang Q and Duan H: Suppressor of cytokine signaling-1 reduces high glucose-induced TGF-betal and fibronectin synthesis in human mesangial cells. FEBS Lett 582: 3484-3488, 2008.

38. Boengler K, Hilfiker-Kleiner D, Drexler H, Heusch G and Schulz R: The myocardial JAK/STAT pathway: from protection to failure. Pharmacol Ther 120: 172-185, 2008 .

39. Calvert JW, Coetzee WA and Lefer DJ: Novel insights into hydrogen sulfide - mediated cytoprotection. Antioxid Redox Signal 12: 1203-1217, 2010.

40. Xu W, Chen J, Lin J, Liu D, Mo L, Pan W, Feng J, Wu W and Zheng D: Exogenous $\mathrm{H}_{2} \mathrm{~S}$ protects $\mathrm{H} 9 \mathrm{c} 2$ cardiac cells against high glucose-induced injury and inflammation by inhibiting the activation of the NF- $\mathrm{NB}$ and IL-1 $\beta$ pathways. Int J Mol Med 35: 177-186, 2015.

41. Xiao T, Luo J, Wu Z, Li F, Zeng O and Yang J: Effects of hydrogen sulfide on myocardial fibrosis and PI3K/AKT1-regulated autophagy in diabetic rats. Mol Med Rep 13: 1765-1773, 2016.

42. Zhou X and Lu X: Hydrogen sulfide inhibits high-glucose-induced apoptosis in neonatal rat cardiomyocytes. Exp Biol Med (Maywood) 238: 370-374, 2013.

This work is licensed under a Creative Commons Attribution-NonCommercial-NoDerivatives 4.0 International (CC BY-NC-ND 4.0) License. 